\title{
e-SUS AB Atividade Coletiva: aplicativo móvel para registro de atividades coletivas em serviços de Atenção Básica
}

\author{
Igor Carvalho Gomes ${ }^{1}$, Ana Claudia Cielo ${ }^{1}$, João Geraldo Oliveira Júnior ${ }^{1}$, Thaísa \\ Cardoso Lacerda ${ }^{2}$
}

${ }^{1}$ Ministério da Saúde, Secretaria de Atenção à Saúde, Departamento de Atenção Básica, Coordenação Geral de Acompanhamento e Avaliação - Estratégia e-SUS Atenção Básica - Brasília - DF - Brazil

${ }^{2}$ Laboratório Bridge, Centro Tecnológico, Universidade Federal de Santa Catarina Florianópolis - SC - Brazil

igor.gomes@saude.gov.br, ana.cielo@saude.gov.br,

joao.geraldo@saude.gov.br, thaisalbridge.ufsc.br

\begin{abstract}
The Primary Health Care teams have the responsibility to identify the most effective and efficient actions to promote and recover the health of the citizens. One way to carry out these actions is carrying out collective activities with the citizens of the territory or with the health care professionals. Collective activities can be carried out in different locations, which are generally not provided with a computer with internet access. In this context a mobile app for tablet called e-SUS AB Atividade Coletiva was developed. The application allows the digitization of the records of collective action in loco reducing the rework of the professionals and facilitating the access to information of previous activities.
\end{abstract}

Resumo. As equipes de Atenção Básica tem a responsabilidade de identificar as ações mais efetivas e eficientes para promover e recuperar a saúde dos cidadãos. Uma das formas de realizar estas ações é por meio das atividades coletivas em saúde. As atividades coletivas podem ser realizadas em diferentes locais, que geralmente não são providos de computador com acesso à internet. Neste contexto, foi desenvolvido o aplicativo para tablet chamado e-SUS AB Atividade Coletiva. $O$ aplicativo permite a digitalização dos registros de atividades de ação coletiva in loco reduzindo o retrabalho dos profissionais e facilitando o acesso a informação de atividades anteriores.

\section{Introdução}

A Atenção Básica (AB) se define como a porta de entrada preferencial do sistema de saúde. Ela deve ordenar os fluxos de pessoas e informações em todos os pontos de atenção à saúde de forma integrada com os demais serviços, de forma a promover um atendimento integral às necessidades de saúde da população [BRASIL, 2017].

As equipes de $\mathrm{AB}$ possuem responsabilidades sobre a saúde de uma população e para tanto devem ser capazes de analisar a realidade de saúde, identificando os meios mais efetivos e eficientes para proteger, promover e recuperar a saúde desta população. Isso significa que os profissionais e gestores devem ser capazes de organizar esses processos, enquanto os sistemas de informação devem apoiar, mapear e classificar os problemas mais prevalentes e que possuem maior impacto sobre a saúde da população. 
A estratégia e-SUS Atenção Básica (e-SUS AB) foi criada pelo Departamento de Atenção Básica do Ministério da Saúde (MS) com intuito de qualificar o registro da informação por meio de software de prontuário eletrônico (PEC - Prontuário Eletrônico do Cidadão). Nessa perspectiva, foram incluídos na Estratégia e-SUS AB aplicativos móveis para registro das ações realizadas pelos profissionais de saúde em locais de difícil manejo de computadores ou notebooks. Assim, o aplicativo e-SUS AB Atividade Coletiva foi desenvolvido para o registro das ações coletivas realizadas pelos profissionais das equipes de Atenção Básica na sua área de abrangência.

As atividades coletivas podem ser realizadas por profissionais de diversos tipos de equipes que atuam na $\mathrm{AB}$ [BRASIL, 2018]. Tais atividades podem ser subdivididas em ações coletivas administrativas e ações coletivas em saúde. As atividades coletivas podem ser realizadas em diferentes locais do território, incluindo escolas, ginásios de esporte, salas de reunião da comunidade, auditórios e a própria unidade de saúde, entre outros. Estes locais geralmente não possuem computador com acesso à internet, dessa forma o registro das atividades é realizado em um formulário de papel (Ficha de Atividade Coletiva) para que possa ser posteriormente digitado no sistema.

Para que estas informações estejam disponíveis para os gestores, é necessário que elas sejam registradas digitalmente e enviadas para o SISAB, por meio do e-SUS AB. Esse registro é realizado no Sistema com Coleta de Dados Simplificada (CDS). O fluxo atual de registro de atividades coletivas ocorre da seguinte forma: 1) Profissionais realizam uma ação coletiva administrativa ou em saúde; 2) Os profissionais registram a ação em uma ficha de papel; 3) Após finalizar a atividade, as informações registradas na ficha de papel são inseridas no Sistema e-SUS AB, pelo próprio profissional, ou por um digitador.

Desde abril de 2013 já foram registradas mais de 15 milhões de atividades coletivas, esse registros representam, de certa maneira, milhões de impressões de fichas de papel. Os problemas do uso de formulários de papel são amplamente discutidos na literatura, entre eles podem ser destacados, impacto ambiental, custos com impressão, aumento do tempo de compartilhamento de informações, custos para armazenamento, maior dificuldade para acessar informações necessárias e consequentemente redução da produtividade [Akunyili, 2015; Adomi, 2010; Onciaiu, 2013; Mukhoryanova, 2016; Iyoha, 2016].

Neste sentido, o aplicativo e-SUS AB Atividade Coletiva apresenta-se como uma alternativa relevante para registro dessas ações em saúde, pois dispensa impressão de fichas e reduz o retrabalho por parte dos profissionais.

\section{2. e-SUS AB Atividade Coletiva}

O aplicativo e-SUS AB Atividade Coletiva foi desenvolvido para utilização em dispositivos do tipo tablet, levando em conta aspectos relacionados ao conforto, à segurança e à usabilidade da ferramenta dentro do processo de trabalho dos profissionais de saúde. Baseado na norma NBR ISO 9241 [ABNT, 2002] foram realizadas entrevistas com profissionais de saúde na qual foi avaliado o aplicativo, por meio de um questionário pós teste com mais de 30 respondentes, com a finalidade de verificar a facilidade de uso, eficiência e satisfação dos usuários.

O aplicativo possibilita a digitalização dos registros de atividades de ação coletiva in loco, levando em consideração o processo de trabalho das equipes AB. Dessa forma, 
elimina-se a necessidade de digitação das informações de atividades coletivas no Sistema CDS. Para que os registros sejam disponibilizados para visualização no PEC e CDS, basta que o profissional faça o envio das informações para a instalação do PEC e-SUS AB, ao retornar para a Unidade Básica de Saúde (UBS). Além disso, o aplicativo permite que os profissionais visualizem informações de atividades coletivas anteriores, uma vez que ele possui uma listagem com as atividades realizadas na UBS nos últimos 3 meses.

Dessa forma, o aplicativo possui dois blocos de atuação: 1) Registro de ações coletivas em saúde voltadas para a população da área de abrangência do território da Unidade Básica de Saúde e 2) Registros de ações coletivas para a organização do processo de trabalho, tendo em vista, as necessidades e situação de saúde do território sob sua responsabilidade. Além disso, as ações realizadas no Programa Saúde na Escola (PSE) e no Programa Nacional de Controle do Tabagismo (PNCT), também podem ser registradas nesta aplicação.

Neste contexto, o fluxo de registro de atividades coletivas utilizando o aplicativo ocorre da seguinte forma: 1) O profissional responsável pela atividade coletiva sincroniza o aplicativo Atividade Coletiva com servidor do PEC e-SUS AB; 2) O profissional registra as informações da ação coletiva no aplicativo durante a atividade; 3) Após finalizar a atividade e retornar à UBS, o profissional realiza novamente a sincronização com o servidor.

\subsection{Arquitetura}

A figura 1 apresenta a arquitetura de alto nível da integração entre aplicativo e-SUS AB Atividade Coletiva com o PEC e-SUS AB. O aplicativo pode ser utilizado por mais de um profissional da $\mathrm{AB}$, desde que possua permissão para registrar fichas de atividade coletiva e que tenha um login e senha de acesso ao PEC e-SUS AB. Para iniciar o uso do aplicativo é necessário realizar uma sincronização com o PEC, que enviará os dados de acesso dos profissionais, dados de cidadãos cadastrados no território e dados de atividades coletivas registradas na UBS nos últimos 3 meses.

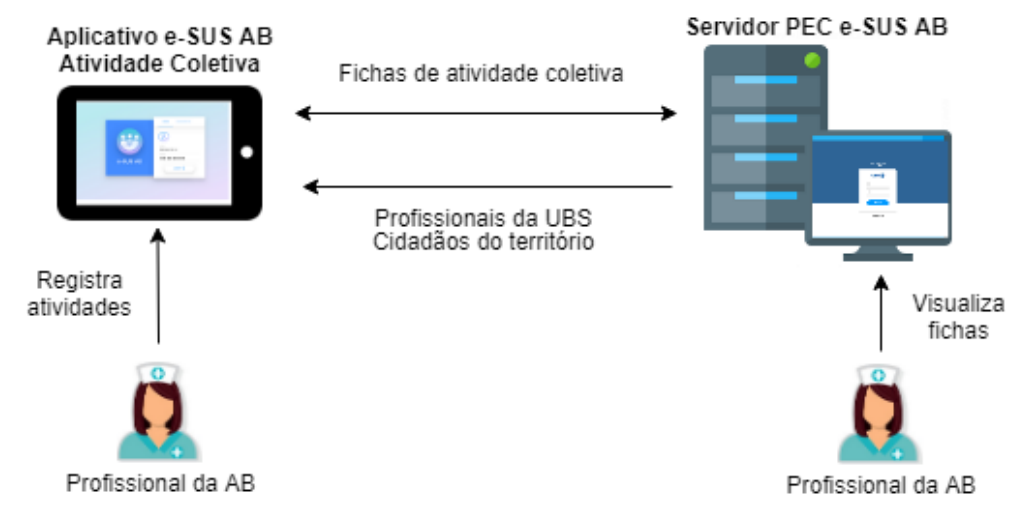

Figura 1. Arquitetura do e-SUS AB Atividade Coletivo

Após a sincronização, o profissional poderá visualizar o registro de atividades anteriores realizadas na UBS, na qual ele possui lotação, bem como realizar o registro de novas atividades. Ao sincronizar novamente com o PEC, serão recebidos cadastros novos ou atualizados de profissionais e cidadãos. Além disso, durante a sincronização ocorre o processo de geração das fichas no formato Thrift. É criada uma ficha para cada prática 
registrada nas atividades coletivas. As atividades enviadas podem ser visualizadas no Sistema CDS.

\subsection{Funcionalidades}

- Visualização de atividades coletivas: apresenta uma listagem com as atividades coletivas realizadas na UBS nos últimos 3 meses e com as atividades que foram cadastradas no aplicativo, mas ainda não foram enviadas para o PEC e-SUS AB.

- Criação de grupos de atividade coletiva: permite criar um agrupamento de atividades coletivas que possuem o mesmo propósito e são realizadas com o mesmo conjunto de cidadãos ou profissionais (ex.: grupo de ginásticas, grupo de idosos) [BRASIL, 2018].

- Registro de atividade coletiva: atividades de ação em saúde e atividades de reunião apresentam um cabeçalho comum, que permite informar: data, turno, número de participantes, local da atividade e se ela pertence ao PSE (Figura 2). O aplicativo permite registrar os profissionais que participaram da atividade, indicando qual deles é o responsável. O aplicativo apresenta uma lista de todos os profissionais lotados na UBS do usuário (Figura 2). Ao finalizar o preenchimento das atividades é apresentada uma tela de revisão, para que as informações sejam revisadas antes de estarem aptas a serem enviadas para o PEC e-SUS AB.

- Registro de atividade de ação em saúde: apresenta campos para informar o Tipo da atividade, Tema em saúde, Práticas em saúde e Público-alvo. É possível informar os cidadãos participantes da atividade selecionando em uma listagem com os indivíduos cadastrados na área da UBS do usuário, ou adicionados manualmente na base do aplicativo. Se forem informadas ações de avaliação ou procedimento coletivo, o profissional poderá informar para cada uma delas se ocorreu avaliação alterada para algum cidadão. Se for informada a prática Antropometria, o aplicativo apresentará campos para preenchimento de Peso e Altura, de cada cidadão apresentando o IMC calculado juntamente com a sua classificação, de acordo com a faixa-etária do cidadão.

- Registro de atividade de reunião: apresenta campos para informar o Tipo da reunião e Temas para saúde.

- Filtro de atividades: para facilitar a visualização dos registros de atividade coletiva, é possível filtrar a listagem de atividades por Tipo, Tema em saúde, Prática e Público-alvo.

- Sincronização com PEC e-SUS AB: permite fazer o envio das informações das atividades coletivas registradas no aplicativo, bem como receber novas atividades que foram registradas no Sistema CDS ou em outros tablets. Durante a sincronização o aplicativo recebe o cadastro de novos profissionais e novos cidadãos cadastrados na UBS (Figura 3). É possível sincronizar o cadastro de profissionais de outras UBS do município, se necessário. 

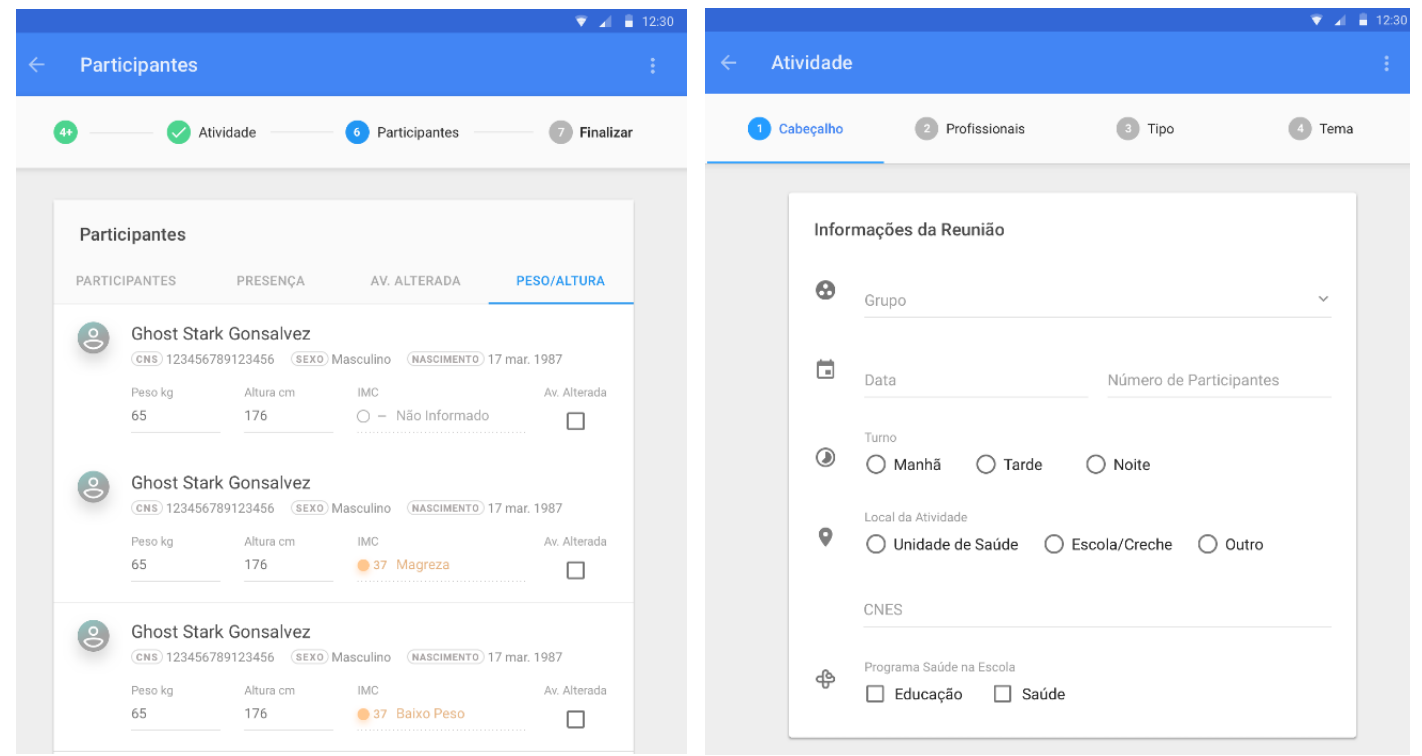

Figura 2. Telas de Cabeçalho e Listagem de Participantes

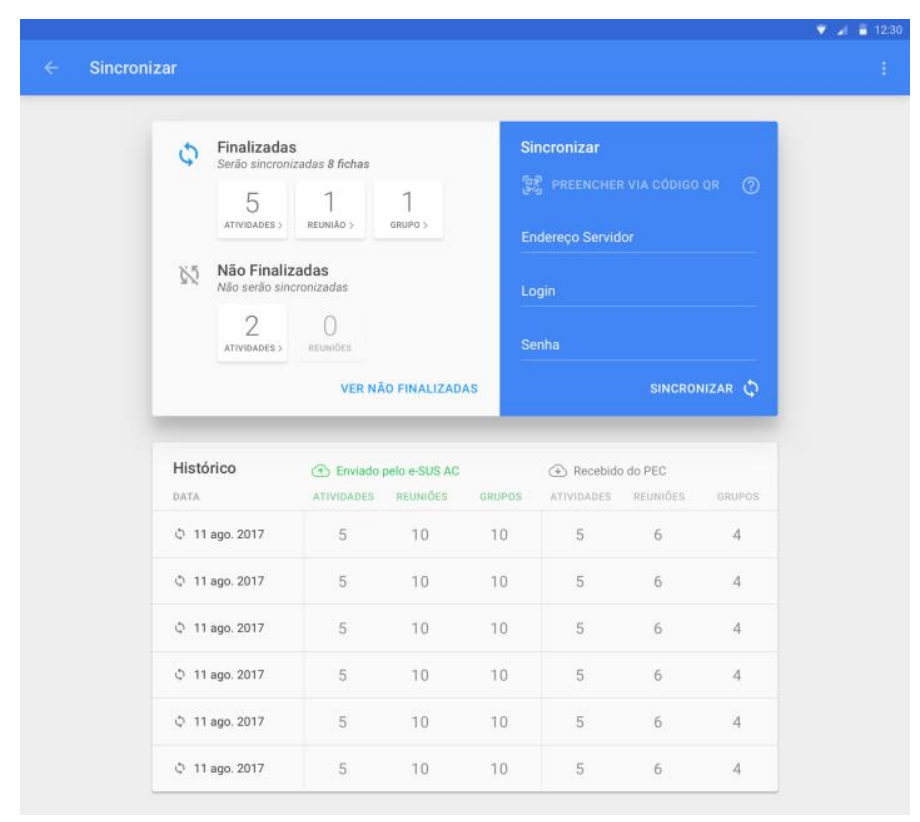

Figura 3. Tela de sincronização

\subsection{Descrição de demonstração}

A demonstração, disponível no link https://bit.ly/2Ur8dhF, apresenta as principais funcionalidades do aplicativo e-SUS AB Atividade Coletiva. É apresentado o processo de sincronização com o Sistema PEC, para obter os dados de lotações profissionais e atividades já registradas. Para conseguir realizar a sincronização é necessário ter usuário no PEC. Em seguida, é mostrado como criar grupos de atividades, como criar duas atividades coletivas em saúde, sendo uma delas, de Educação em grupo e outra de Avaliação/Procedimento coletivo. A demonstração apresenta também como registrar atividades de reunião e como salvar atividades em modo rascunho, para finalizar o preenchimento posteriormente. É apresentado como resolver inconsistências de 
preenchimento das atividades e como utilizar o filtro de atividades. Por fim, apresentamos como realizar o envio das fichas registradas por meio da sincronização com o PEC.

\section{Conclusão}

A solução aqui apresentada possui grande potencial para facilitar o processo de trabalho dos profissionais de saúde da AB. Entende-se que ferramentas tecnológicas, disponibilizadas para esses profissionais, podem apoiar de maneira importante no dia-adia. O aplicativo e-SUS AB Atividade Coletiva cumpre um papel relevante no que tange às ações coletivas, que ocorrem com bastante frequência nas UBS de todo o país.

Limitações em relação a ausência de conectividade local e sincronização manual ainda são duas situações a serem evoluídas. Os principais benefícios podem ser listados com o uso do aplicativo, tais como, a diminuição ou eliminação das fichas em papel e sua digitação, redução de armazenamento físico destes registros, maior eficiência no compartilhamento das informações com todos os membros da equipe, mais facilidade no registro da informação, dentre outros. As avaliações de usabilidade devem ser mantidas para ganho de maturidade do aplicativo. Tudo isso contribui para a melhoria da qualidade e frequência dos registros de atividades coletivas na $\mathrm{AB}$.

\section{Referências}

Adomi, E. (2010). Frameworks for ICT Policy: Government, Social and Legal Issues: Government, Social and Legal Issues. IGI Global.

Akunyili, D. (2015) ICT and e-government in Nigeria. Nkuphe,: A revelation on Africa. Access on, v. 5, p. 10-16.

Abnt (2002). NBR ISO 9241: ergonomia da interação humano-sistema. Rio de Janeiro: ABNT.

Brasil (2017). Portaria No 2.436, de 21 de setembro 2017. Aprova a Política Nacional de Atenção Básica, estabelecendo a revisão de diretrizes para a organização da Atenção Básica, no âmbito do Sistema Único de Saúde (SUS). Brasília.

Brasil (2018). Ministério da Saúde. Secretaria de Atenção à Saúde. e-SUS Atenção Básica: Manual do Sistema com Prontuário Eletrônico do Cidadão PEC - Versão 3.1 [recurso eletrônico] / Ministério da Saúde, Secretaria de Atenção à Saúde, SecretariaExecutiva. - Brasília: Ministério da Saúde.

Brasil (2018). Ministério da Saúde. Secretaria de Atenção à Saúde. e-SUS Atenção Básica : Manual de Uso do Aplicativo e-SUS AB Atividade Coletiva - Versão 1.0 [recurso eletrônico] / Ministério da Saúde, Secretaria de Atenção à Saúde, Departamento de Atenção Básica. - Brasília: Ministério da Saúde.

Mukhoryanova, O. et al (2016). E-Government in the Western European Countries, Asia and in the USA. Indian Journal of Science and Technology, v. 9, n. 16, p. 9075790757.

Iyoha, F. et al (2016). E-government adoption and environmental bonuses: A study of nigeria and United Kingdom. In: European Conference on e-Government. Academic Conferences International Limited. p. 91.

Oncioiu, I. (2013). Business innovation, development, and advancement in the digital economy. IGI Global. 